\title{
MiR-9-5p promotes rabbit preadipocyte differentiation by suppressing leptin gene expression
}

\section{Gang Luo}

Sichuan Agriculture University

\section{Shenqiang $\mathrm{Hu}$}

Sichuan Agricultural University

Tianfu Lai

Sichuan Agricultural University

Jie Wang

Sichuan Agricultural University

Li Wang

Sichuan Agricultural University

Songjia Lai ( $\square$ laisj5794@163.com )

Sichuan Agricultural University

\section{Research}

Keywords: MiR-9-5p, Rabbits, Preadipocyte, Differentiation, Leptin

Posted Date: January 16th, 2020

DOI: https://doi.org/10.21203/rs.2.21007/v1

License: (1) This work is licensed under a Creative Commons Attribution 4.0 International License.

Read Full License

Version of Record: A version of this preprint was published at Lipids in Health and Disease on June 5th, 2020. See the published version at https://doi.org/10.1186/s12944-020-01294-8. 


\section{Abstract}

Background: MicroRNAs are a class of small non-coding RNAs and participate in the regulation of cell differentiation programs. Previous studies have demonstrated that miR-9-5p play a key role during cancer cell research, but the mechanisms regulating of miR-9-5p in adipogenesis remain poorly understood. This study intended to investigate its significance in rabbits with high quality meat by observing the regulating effect of miR-9-5p in pre-adipocytes and finding related targets.

Methods: In this study, a dual luciferase reporter assay was employed to validate the targeting relationship between miR-9-5p and leptin gene. We also utilized quantitative reverse transcription PCR (qRT-PCR), western blot, determination of triglyceride and oil red $O$ staining assay to analyze the regulation of miR-9-5p and leptin gene during adipocyte differentiation.

Results: The analysis demonstrated that during pre-adipocyte differentiation, miR-9-5p was up-regulated and the fat formation related biomarkers fatty acid-binding protein 4(FABP4), CCAAT-enhancer binding protein a $(C / E B P a)$, and peroxisome proliferator activated receptory (PPARY) were also up-regulated. Meanwhile, the oil red $O$ staining assay revealed that the accumulation of lipid droplets increased. We explored the expression pattern and role of miR-9-5p in adipogenesis using white pre-adipocytes. The results showed that miR-9-5p was up-regulated during pre-adipocyte differentiation and overexpression of miR-9-5P enhanced lipid accumulation. Furthermore, we found overexpression of miR-9-5p significantly up regulated the mRNA levels of marker gene PPARY, C/EBPa and FABP4, as well as the protein levels of $P P A R Y$ and content of triglyceride, the results suggested that miR-9-5p might be involved in the regulation of rabbit pre-adipocyte differentiation. We predicted leptin is a target of miR-9-5p by using bioinformatics tools and the conclusion was validated in a luciferase reporter assay. Finally, we verified inhibition of leptin by si-leptin promoted rabbits pre-adipocyte differentiation.

Conclusion: In a word, these results indicate that miR-9-5p promotes rabbits white pre-adipocyte differentiation by targeting leptin .

\section{Introduction}

Adipose tissues, which may be brown, beige or white, not only maintain energy metabolism by generating heat or storing lipids but also serve as an endocrine organ. Of them, white adipose tissues are widely available and have the ability to differentiate. Adipose tissue-derived stem cells (ASCs) are initially recruited by certain stimulation, followed by being transformed into preadipocytes, and are ultimately developed into mature adipocytes(Francisco et al. 2016). Adipogenesis, a complex process in which fibroblast-like pre-adipocytes differentiate into lipid-laden and insulin-responsive mature adipocytes(Lefterova and Lazar 2009), requires sequential activation of numerous transcription factors and noncoding RNAs. During adipogenesis, several transcription factors such as peroxisome proliferatoractivated receptor-y (PPARY) and members of the CCAAT/enhancer-binding family of proteins (C/EBPa) participate in regulating adipogenesis(Hamam et al. 2014; Prayitno et al. 1997). Expression of FABP4 is 
induced during adipocyte differentiation and is controlled by insulin and peroxisome proliferatoractivated receptor $y$ agonists(Hunt et al.). Hyperinsulinism or insensitivity of leptin can lead to the formation of positive feed-back between insulin and leptin, which further increases adipose tissue and content of leptin(Brüning et al. 2000; Wang et al. 2001). Leptin plays a pivotal role in regulating feed intake, energy expenditure and fat deposition in mammals(Matteri 1998). Leptin can promote lipolysis and thermogenesis by influencing the sympathetic nervous system(Eikelis and N. 2004). In addition, leptin can also directly regulate metabolism of some tissues and cells(McClelland et al. 2004; Sanigorski et al. 2013). Leptin resulted in a dose and time-dependent increase in type1 collagen, a decrease of adipsin and leptin mRNA levels, and a 50\% decrease in lipid droplet formation(Thierry et al. 1999). Adenovirus-induced hyperleptinemia in normal rats caused fat loss, downregulation of lipogenic enzymes, and suppression of the transcription factor peroxisome proliferator-associated receptorY(PPARY)(Zhou et al. 1999). The absence of fat in transgenic mice was associated with a 20 -fold reduction in leptin levels and led to marked metabolic alterations including hyperlipidemia, insulin resistance, and diabetes(Moitra et al. 1998). Research recently showed that leptin enhanced differentiation of osteoblasts and inhibited differentiation of adipocytes in human bone marrow stromal cells (Thierry et al. 1999). Taken together, these finding showed that leptin may act locally in adipose tissue to influence the formation of mature adipocytes.

Furthermore, microRNAs, as a class of short noncoding RNA, are generally regarded to inhibit target gene expression by binding to their 3 '-UTRs and have been demonstrated to regulate lipid metabolism(Bagga et al. 2005; Lagos-Quintana and M. 2001). miRNAs were also shown to be involved in cell differentiation(Zhang et al. 2017). In goats, miR-183 promoted preadipocyte differentiation by suppressing expression of Smad4(Zhao et al. 2018) and miR-21a-5p induced pre-adipocyte differentiation by targeting Mitogen-activated protein kinase 2/ kinase 3 (MAPK2K3) through MKK3/p38/MAPK in mice(Xie et al. 2016). However, miR-33b downregulated the differentiation of porcine preadipocytes (Taniguchi et al. 2014). In 3T3-L1 cells, miR-26b promoted adipocyte differentiation through targeting PTEN(Li et al. 2017), while one study has demonstrated that miR-148a-3p impaird rabbit adipocyte differentiation by targeting PTEN in rabbits preadipocytes (He et al. 2018). MiR-9-5p is a well-conserved microRNA and primarily expresses in the central nervous system(Kapsimali et al. 2007; Wienholds and E.). Another study also showed that miR-9-5p can promote MSC (mesenchymal stem cells) migration by activating $\beta$-catenin signaling pathway (Li $X$ et al. 2017). $\beta$-catenin is an integral part of Wnt signaling and play an important role in Wnt signaling. The study has found that the classical Wnt signal can inhibit fat differentiation(Ross et al. 2000). It has also been shown that C/EBPa or PPARy can inhibit Wnt signal, which can affect the differentiation of adipocytes by reducing the level of $\beta$ catenin(MOLDES et al. 2003). Yet the role of miR-9-5p in differentiation of adipocytes remains unknown.

As an economically important animal and an animal model, adipose tissue deposition can not only affect intramuscular fat content, but also can cause obesity. Intramuscular fat is associated with juiciness and flavor(Warner et al. 2010) and the importance of intramuscular fat (IMF) content on meat quality is well established(Warriss 1999; Wood et al. 2008). Therefore, it is necessary to study the regulatory mechanism of adipocytes. In this study, we isolated and cultured rabbit perirenal adipose cells. Then, we 
studied the effect of miR-9-5p on adipocyte differentiation by overexpression and inhibition of miR-9-5p expression. In addition, we also predicted and verified that miR-9-5p is a target gene of leptin. Finally, we found miR-9-5p can influence differentiation of adipocyte by downregulating the mRNA expression levels of leptin.

\section{Materials And Methods}

\subsection{Animals and tissues collection}

All the experimental procedures using rabbits in this study were conducted under a protocol approved by the Institutional Animal Care and Use Committee in the College of Animal Science and Technology, Sichuan Agricultural University, China. Perirenal adipose tissues were collected from three newborn Zealand rabbits. which were raised under standard condition at the Sichuan Agricultural University farm (Yaan, Sichuan, China).

\subsection{Cell isolation, culture and induction of adipogenesis}

Adipose tissue was rinsed three times in PBS then minced and digested with $0.25 \%$ collagenase type I (Gibco, Carlsbad, CA, USA) at $37^{\circ} \mathrm{C}$ for $1 \mathrm{~h}$. The mixture was mixed with complete medium (CM; DM/F12, $10 \%$ fetal bovine serum, $2 \%$ penicillin-streptomycin) (Gibco) followed by filtration through 70 - and 40 -nm cell sieve, respectively. Finally, the mixture solution was centrifuged at $1200 \times \mathrm{g}$ for $5 \mathrm{~min}$ to collect the preadipocytes. The preadipocytes were seeded in culture flask with complete medium and the flask was placed at $37^{\circ} \mathrm{C}$ in a humidified incubator with $5 \% \mathrm{CO}_{2}$. Culture medium was changed every 2 days and the cell was frozen for future studies when they were passed to the third generation. To induce differentiation, an adipogenic inducer cocktail (0.5 mM 3-isobutyl-1-methylxanthine, 10\% FBS, $1 \mu \mathrm{M}$ dexamethasone, and $1.7 \mu \mathrm{M}$ insulin)was added into the growth medium when cell density reached about $70 \%$ confluence and the cells were subsequently cultured for a further $72 \mathrm{~h}$, after which the medium was replaced with maintenance medium (growth medium supplemented with $1.7 \mathrm{mM}$ insulin per $50 \mathrm{~mL}$ ) for an additional $72 \mathrm{~h}$. Thereafter, the cells were cultured in growth medium until maturation at ten days.

\subsection{Cell transfection}

For transfection, preadipocyte cells were seeded into 24-well or 6-well plates and transfected using Lipofectamine 3000 (Invitrogen, Carlsbad, CA, USA) according to the manufacturer's instructions after the cells had reached $70 \%$ confluency. The final concentrations of the miRNA mi-mics (mimics NC), inhibitor (inhibitor NC), and leptin siRNA (siRNA NC) were $50 \mathrm{nM}, 100 \mathrm{nM}$, and $100 \mathrm{nM}$, respectively. Three independent repetitions were performed for each treatment group. The cells were harvested at different time after transfection and used to study adipogenic differentiation.

\subsection{RNA isolation and $q P C R$ analysis}

Total cellular RNA was isolated with RNAiso Plus reagent (TaKaRa, Shiga, Japan) according to the manufacturer's instructions. RNA purity was determined by OD $260 \mathrm{~nm} /$ OD $280 \mathrm{~nm}$ and OD $260 \mathrm{~nm} /$ OD 
$230 \mathrm{~nm}$ ratios with the Nano Drop 2000 UV-Vis Spectrophotometer (Thermo Scientific, Waltham, MA, USA). First-strand CDNA of total RNA and small RNA was synthesized using the Prime Script RT reagent Kit (Takara, Japan) and the SYBR ${ }^{\circledR}$ Prime Script ${ }^{\text {TM }}$ miRNA reverse transcription Kit (Takara), respectively, according to the manufacturer's protocol. The corresponding CDNA was stored at $-20^{\circ} \mathrm{C}$. qPCR used the SYBR premix Ex Taq II (Tli RNase H Plus) (Catalog No. RR820A; Takara) on a CFX96 system (Bio-Rad, Hercules, CA, USA). U6 was selected to normalize the expression of miRNA, while the internal reference used for mRNA expression was actin. The $2^{-\Delta \Delta C t}$ method was used to analyze the relative expression of each gene. The sequences of the primers used for QPCR are shown in Table 1. 
Table 1

Primers used in this study

\begin{tabular}{|c|c|c|c|}
\hline Gene name & Primer sequence (5'-3') & $\left(\mathrm{Tm} /{ }^{\circ} \mathrm{C}\right)$ & (Product size/bp) \\
\hline \multirow[t]{2}{*}{ leptin } & CTGTGCCCATGCGGAAAGTC & 61.4 & 104 \\
\hline & AGTCCAAACCGACGACCCTC & & \\
\hline \multirow[t]{2}{*}{$\beta$-actin } & GGAGATCGTGCGGGACAT & 61.4 & 318 \\
\hline & GTTGAAGGTGGTCTCGTGGAT & & \\
\hline \multirow[t]{2}{*}{ PPARY } & GAGGACATCCAGGACAACC & 61 & 168 \\
\hline & GTCCGTCTCCGTCTTCTTT & & \\
\hline \multirow[t]{2}{*}{ FABP4 } & GGCCAGGAATTTGATGAAGTC & 61.4 & 140 \\
\hline & AGTTTATCGCCCTCCCGTT & & \\
\hline \multirow[t]{2}{*}{$\mathrm{C} / \mathrm{EBPa}$} & GCGGGAACGAACAACAT & 64 & 172 \\
\hline & GGCGGTCATTGTCACTGGTC & & \\
\hline miR-9-5p mimic & $\begin{array}{l}\text { UCUUUGGUUAUCUAGCUGUAUGA } \\
\text { AUACAGCUAGAUAACCAAAGAUU }\end{array}$ & & \\
\hline Negative Control (NC) & $\begin{array}{l}\text { UUCUCCGAACGUGUCACGUTT } \\
\text { ACGUGACACGUUCGGAGAATT }\end{array}$ & & \\
\hline inhibitor-9-5p & UCAUACAGCUAGAUAACCAAAGA & & \\
\hline inhibitor NC & CAGUACUUUUGUGUAGUACAA & & \\
\hline si-leptin-1 & $\begin{array}{l}\text { CCACAAUGGACCAGACGUUTT } \\
\text { AACGUCUGGUCCAUUGUGGTT }\end{array}$ & & \\
\hline si-leptin-2 & $\begin{array}{l}\text { CCAUUGUCACCAGGAUCAGTT } \\
\text { CUGAUCCUGGUGACAAUGGTT }\end{array}$ & & \\
\hline si-leptin-3 & $\begin{array}{l}\text { CCGAAAUGUGAUCCAAAUATT } \\
\text { UAUUUGGAUCACAUUUCGGTT }\end{array}$ & & \\
\hline Primers for gene 3'UTR & ATGGGTAAGTACATCAAGAG & & \\
\hline cloning leptin & GAGGTCCGAAGACTCATTT & & \\
\hline
\end{tabular}

\subsection{Western blotting}

Total protein of cell samples was extracted with the Total Protein Extraction Kit (Sangon, Shanghai, China) according to the manufacturer's protocol and protein content was quantified with the BCA Protein Assay Kit. Forty micrograms of proteins were resolved by $8 \%-12 \%$ SDS-polyacrylamide gels and transferred to a PVDF membrane (Bio-Rad). The PVDF membrane was rinsed with TBS-Tween20 (TBST) and blocked for $2 \mathrm{~h}$ in skimmed milk. Then, the membranes were incubated with anti-PPARY (Santa Cruz 
Biotechnology, and anti- $\beta$-actin (Abs, Beijing, China) according to the respective instructions overnight. the PVDF membranes were incubated with the secondary goat anti-mouse IgG $(H+L)$ (Transgen, IllkirchGraffenstaden France) for $2 \mathrm{~h}$ after being washed three times. The protein bands were incubated with chemiluminescence reagents (Beyotime) after being washed four times. The images were obtained with a Bio-Rad GelDoc system equipped with a Universal Hood III (Bio-Rad), and the integrated optical density (IOD) was calculated using Gel-Pro Analyzer 4.0.0.4. Actin was used as an internal control.

\subsection{Oil Red $O$ staining and determination of triglyceride content}

Adipocytes were washed three times with phosphate-buffered saline (PBS) and fixed with $4 \%$ paraformaldehyde for 30 min. After being washed three times with PBS, the cells were stained with $1 \%$ filtered oil red-O solution for about $30 \mathrm{~min}$ in the dark. Then, adipocytes were washed and observed using a phase contrast microscope. Finally, oil Red $O$ was eluted from the stained cells with isopropanol and quantified by measuring the optical density values at $510 \mathrm{~nm}$ wavelength. Intracellular triglyceride (TG) content was quantified with the TG Assay Kit (Applygen, Beijing, China) according to the manufacturer's protocol. The TG content ( $\mathrm{nmol} / \mathrm{mg}$ protein) was normalized to the cellular protein concentration, and the protein concentrations were measured with the BCA Protein Assay Kit (Beyotime, Shanghai, China).

\subsection{Prediction and verification of the target gene of miR-9- $5 \mathrm{P}$}

MiRwalk (http://zmf.umm.uni-heidelberg.de/apps/zmf/mirwalk2/) was employed to predict the target genes of miR-9-5p and leptin was the target gene of miR-9-5p. The 3'UTR of leptin containing the miR-9$5 p$ target site was cloned into the Sac I-Xba I site of the pmirGLO Vector (Promega,Madison, WI) to construct luciferase reporter plasmids. The primers used for plasmid construction are listed in Table 1, and multiple cloning sites were located downstream of firefly luciferase gene. Hela cells were seeded into 24-well plates in triplicate. Then, the pmirGLO-leptin-3' UTR of wild or mutant type was co-transfected with the synthetic miR-9-5p mimic into Hela cells after the cell density reached $70-80 \%$. Firefly luciferase (luc2) activity was measured $48 \mathrm{~h}$ after transfection and normalized to Renilla luciferase activity according to the TransDetect ${ }^{\circledR}$ Doubl Luciferase Reporter Assay Kit instructions (Transgen, Beijing, China).

\subsection{Statistical analysis}

Data were analyzed by GraphPad Prism7(GraphPad Software, La Jolla, CA, USA), and the results are presented as the mean $\pm S E$ based on at least three independent experiments. $P<0.05$ and $P<0.01$ were deemed to be significant and highly significant, respectively.

\section{Results}




\subsection{Expression profiling of miR-9-5p during rabbit preadipocyte differentiation}

In the process of inducing differentiation, the adipocytes were fully differentiated, depositing large lipid droplets (Fig. 1A). Results of oil red-O staining showed that lipid droplets rapidly increased during preadipocyte cell differentiation (Fig. 1B). Simultaneously, the expression of miR-9-5p grew rapidly after three days of induction and the expression on the ninth day was significantly higher than that on the other days (Fig. 1C). Correspondingly, Adipogenic marker gene FABP4 had the highest expression on the sixth day whereas PPARY and CEBPa had the highest expression on the third day after induction (Fig. 1D, E, F).

\subsection{Upregulation of miR-9-5p promotes rabbit preadipocyte differentiation}

To investigate the function of miR-9-5p in rabbit preadipocyte cell differentiation, we first detected the expression level of miR-9-5p in the cells transfected with miR-9-5p mimics and NC during differentiation and the miR-9-5p showed a higher expression level in the mimic group than that of the control after induction $(P<0.01)$ (Fig. 2A). We found lipid accumulation was more in mimic group than the control by oil red-O staining and quantitative detection of oil red $O$ staining $(P<0.01)$ (Fig. 4B, C). In addition, $A$ significant increased in triglyceride after overexpression of miR-9-5p (Fig. 2D). The mRNA levels of adipogenic markers including PPARY and C/EBPa were higher significantly in the mimic group than NC group on the first and third days $(P<0.01)$ (Fig. 2E, F, G). Western blot analysis further indicated that PPARY protein levels were higher significantly in the mimic group than NC group after two days of transfection $(P<0.05)($ Fig. $2 \mathrm{H}, \mathrm{I})$.

\subsection{Downregulation of miR-9-5p inhibited rabbit preadipocyte differentiation}

We next examined differentiation of preadipocytes when endogenous miR-9-5p was effectively inhibited with the miR-9-5p inhibitor. As shown in Figure 3A, the expression levels in the inhibitor groups were lower significantly than that of the control on the 3rd, 5th, 7th and 9th day after transfection $(P<0.01)$. We found that down-regulation of miR-9-5p decrease lipid accumulation in the preadipocytes cells as detected by Oil Red $\mathrm{O}$ staining (Fig. 3B, C) and TG content (Fig. 3D). The mRNA expression levels of the common differentiation-related genes (PPARY and $C / E B P a)$ on the 1st and 3rd day after transfection had lower levels of expression in the inhibition groups(Fig. 3E, F), while the common differentiation-related genes FABP4 on the 5th and 7th day after transfection had lower levels of expression in the inhibition groups $(P<0.01)$ (Fig. 3G). In addition, PPARY protein expression level decreased after miR-9-5p inhibition (Fig. 3H, I). 


\subsection{Leptin is a target gene of miR-9-5p}

To further determine the function of miR-9-5p, bioinformatics analysis was carried out to predict direct targets of miR-9-5p. MiRWalk2.0 (http://zmf.umm.uni-heidelberg.de/apps/zmf/mirwalk2/) software revealed that leptin contains the target sites for miR-9-5P (Fig. 4A). To verify existence of the direct binding between miR-9-5p and leptin, the luciferase reporter assay showed the firefly luciferase activity significantly reduced during cotransfection of pmirGLO-LEPTIN-3' UTR and miR-9-5p agomirs (Fig. 4B).

\subsection{MiR-9-5p downregulated leptin mRNA expression}

To better understand the mechanism of miR-9-5p during preadipocyte differentiation, we further validated the effect of miR-9-5p on the expression of leptin. As shown in Figure 5A, overexpression of miR-9-5p led to an obvious decrease in the mRNA levels of leptin. In contrast, when the endogenous miR-9-5p was inhibited with the miR-9-5p inhabitor, the expression level of leptin was increased, compared with the INC group (Fig. 5B).

\subsection{Downregulation of leptin expression promoted rabbit preadipocyte differentiation}

To further confirm whether leptin is involved in the differentiation of rabbit preadipocytes, si-leptin was utilized to knockdown endogenous leptin. We found lipid accumulation and accumulation of triacylglycerol accelerated in the preadipocytes cells (Fig. 6A, B, C). When leptin was knocked down, the mRNA levels of PPARY and C/EBPa rapidly increased on the 1 st and 3rd day after transfection, whereas FABP4 rapidly increased on the 5th and 7th (Fig. 6D, E, F). Furthermore, PPARY protein expression level was higher significantly in si-leptin group than NC group after transfection 3 days $(P<0.05)$ (Fig. 6G, H). Additionally, we found the expression level rapidly decreased after knocking down endogenous leptin (Fig. 6I).

\section{Discussion}

In recent years, miRNAs were reported to be expressed in mammalian adipocytes and played an important role in the regulation of adipogenesis(Xu P et al. 2003). Previous studies have discovered a functional interplay among glucose-dependent insulin secretion, mir-9 levels and Sirt1 protein in pancreatic islets b-cells(Ramachandran et al. 2011). It has also been found that miR-9 and Sirt1 are involved in glucose dependent insulin secretion of islet $\beta$ cells, and it has been proved that Sirt1 is a target gene of miR-9(Plaisance et al. 2006b). In addition, miR-9-5p could negatively regulate Sirt1 expression and played an important role in the regulation of cells proliferation and migration(Qi et al. 2015). Sirt1 can bind to two coenzyme factors of PPARy, which inhibits the binding of PPARy to the target gene and the differentiation of adipocytes(Picard et al. 2004). It has also been shown that miR-9- 
$5 p$ promotes the secretion of Granuphilin-a and reduces the secretion and synthesis of insulin by targeting Onecut2(Plaisance et al. 2006a). PPARy can increase insulin sensitivity of adipose tissue through adipose tissue specificity(MOLDES et al. 2003) and PPARY is a marker gene of preadipocyte differentiation. So, miR-9-5p may regulate the differentiation of adipocytes.

We found triglyceride content was more and lipid droplets was larger after transfection of miR-9-5p. The expression levels of adipogenic marker gene FABP4, PPARY and CEBPa increased after over-expression miR-9-5p, while downregulation of miR-9-5p using the synthetic inhibitor markedly reduced the formation of neutral lipid droplets and suppressed the expression of marker genes at both the mRNA and protein levels. These results suggested that miR-9-5p can promote the differentiation of rabbit preadipocytes. Previous studies demonstrated that NADPH oxidase 4 (NOX4) is a target gene of miR-9-5p(FierroFernandez et al. 2015) and NOX4 is the key enzyme of reactive oxygen species (ROS). It was reported that insulin release is inhibited by increasing ROS of islet B cells(Anvari et al. 2015; Den Hartigh et al. 2017) and the content of leptin is positive correlated with insulin (Anderwald et al. 2002; Papaspyrou-Rao et al. 1997). Then, a dual luciferase reporter assay was employed to validate that leptin is a target gene of miR-9-5p. The result suggested miR-9-5p can negatively regulate leptin gene expression. Previous studies have shown miR-9-5p can regulate secretion and synthesis of insulin. Insulin can stimulate secretion of leptin and increase the mRNA expression of leptin, meanwhile, leptin can directly inhibit the secretion of insulin(Emilsson et al. 1997). Studies have shown that insulin can improve the differentiation rate of preadipocytes and promote the accumulation of fat(Sato et al. 1996). It has also been found insulin may affect the expression of PPARy by affecting Sterol regulatory element-binding protein-1c (SREBP-1c) (Garcia-Escobar et al. 2011). Leptin can also regulate differentiation of adipocytes and previous studies have demonstrated leptin can inhibit the synthesis of lipids and promote the decomposition of triglycerides in adipocytes(Flier and Spiegelman 2001). So, miR-9-5p may target leptin to promote the differentiation of rabbit preadipocytes.

Leptin is secreted by adipocytes and the mRNA level of leptin decreased at the later stage of adipocyte differentiation(Ailhaud et al. 1992). Previous studies have been shown that leptin involved in a local ultrashort negative feedback loop and the loop regulated the differentiation of preadipocytes(Thierry et al. 1999). A study has also been shown that leptin suppresses specific biochemical processes, which contribute to lipid accumulation and adipocyte differentiation(Shimabukuro et al. 1997; Zhang and S. 1996). Furthermore, leptin can accelerate the proliferation of preadipocytes, inhibit the synthesis of lipids and promote the decomposition of triglycerides(Flier and Spiegelman 2001). We found the suppressed expression of leptin dramatically promoted the differentiation of preadipocytes. This finding is similar to previous study that leptin inhibits differentiation of preadipocytes. Therefore, we speculated that miR-9$5 p$ positively regulates rabbit preadipocyte differentiation by inhibiting leptin. However, the biological basis and mechanism of this phenomenon need further research to get rabbits of high intramuscular fat content. In recent years,many studies have been done to get animals with high intramuscular fat content in cattles and pigs, but it is little progress. Because we need to reduce body fat and increase muscle fat in the same animal at the same time. However, rabbits are an animal with particularly low-fat content. So, 
we can increase the body fat and intramuscular fat at the same time, which brings hope to cultivate a new kind of rabbit with high intramuscular fat content.

\section{Conclusions}

In summary, our data demonstrated that miR-9-5p can promote the differentiation of rabbit preadipocytes and leptin acts as one of the downstream targets of miR-9-5p. In addition, knockdown of leptin promoted adipogenic differentiation. Taken together, we concluded that miR-9-5p promotes rabbit preadipocyte differentiation by suppressing expression of leptin mRNA.

\section{Abbreviations}

FABP4: fatty acid-binding protein 4; C/EBPa: CCAAT-enhancer binding protein a; PPARY: peroxisome proliferator activated receptory; ASCs: Adipose tissue-derived stem cells; MAPK2K3: Mitogen-activated protein kinase 2/ kinase 3; 3T3-L1: Mouse Embryonic mouse fibroblast; PBS: phosphate-buffered saline; NOX4: NADPH oxidase 4; ROS: reactive oxygen species; SREBP-1c: Sterol regulatory element-binding protein-1c.

\section{Declarations}

\section{Acknowledgements}

We thank the staff at our laboratory for their ongoing assistance.

\section{Authors' contributions}

GL, JW, SJL designed and directed the study. GL, TFL, LW performed the experiments, data analysis and drafted the manuscript. GL contributed to the analysis and writing of the manuscript. QSH critically reviewed drafts of the manuscript and made comments to improve clarity. All authors approved the final version of this article.

\section{Funding}

This work was supported by National rabbit industry technology system (CARS-43-A-2).

\section{Availability of data and materials}

All data generated or analyzed during this study are included in this published article.

\section{Animal ethical approval}

All experimental operations are carried out in strict accordance with the Experimental Animal Operation Regulations (No.18 [2014] of Sichuan Agricultural University), which formulated by the Sichuan Agricultural University experimental animal operation regulations and welfare management committee. 
Surgical procedures involving rabbits were performed according to the approved protocols of the Biological Studies Animal Care and Use Committee, Sichuan Province, China. Rabbits had free access to food and water under normal conditions and were humanely sacrificed as necessary to ameliorate suffering.

\section{Consent for publication}

Not applicable.

\section{Competing interests}

The authors declare that they have no competing interests.

\section{Author details}

${ }^{1}$ Animal Genetic Resources Exploration and Innovation Key Laboratory of Sichuan Province, Sichuan Agricultural University, Chengdu 611130, China.

\section{References}

Ailhaud G, Grimaldi P, A , R N (1992) Cellular and Molecular Aspects of Adipose Tissue Development Annual Review of Nutrition 12:207-233

Anderwald C, Müller G, Koca G, Fürnsinn C, Waldh usI W, Roden M (2002) Short-Term Leptin-Dependent Inhibition of Hepatic Gluconeogenesis Is Mediated by Insulin Receptor Substrate-2 Molecular Endocrinology 16:1612-1628

Anvari E, Wikstrm P, Walum E, Welsh N (2015) The novel NADPH oxidase 4 inhibitor GLX351322 counteracts glucose intolerance in high-fat diet-treated C57BL/6 mice Free Radical Research 49:1-32

Bagga S, Bracht J, Hunter S, Massirer K, Holtz J, Eachus R, Pasquinelli AE (2005) Regulation by let-7 and lin-4 miRNAs Results in Target mRNA Degradation Cell 122:553-563

Brüning JC, Gautam D, Burks DJ, Gillette J, Kahn CR (2000) Role of Brain Insulin Receptor in Control of Body Weight and Reproduction Science 289:2122-2125

Den Hartigh LJ, Omer M, Goodspeed L, Wang S, Wietecha T, O’Brien KD, Han CY (2017) Adipocyte-specific deficiency of NADPH oxidase 4 delays the onset of insulin resistance and attenuates adipose tissue inflammation in obesity Arteriosclerosis, thrombosis, and vascular biology 37:466-475

Eikelis, N. (2004) Extra-adipocyte leptin release in human obesity and its relation to sympathoadrenal function AJP: Endocrinology and Metabolism 286:E744-E752 
Emilsson V, Liu Y-L, Cawthorne MA, Morton NM, Davenport M (1997) Expression of the Functional Leptin Receptor mRNA in Pancreatic Islets and Direct Inhibitory Action of Leptin on Insulin Secretion Diabetes 46:313-316

Fierro-Fernandez M et al. (2015) miR-9-5p suppresses pro-fibrogenic transformation of fibroblasts and prevents organ fibrosis by targeting NOX4 and TGFBR2 Embo Reports 16:1358-1377

Flier J, Spiegelman GB (2001) Obesity and the regulation of energy balance Cell 104:531-543

Francisco R-O, Azahara R, Carolina G-L, Angel G, Concepción A (2016) Cell Models and Their Application for Studying Adipogenic Differentiation in Relation to Obesity: A Review International Journal of Molecular Sciences 17:1040-1066

Garcia-Escobar E, Rodriguez-Pacheco F, Haro-Mora J, J (2011) Effect of insulin analogues on 3t3-11 adipogenesis and lipolysis European Journal of Clinical Investigation 41:979-986

Hamam D, Ali D, Kassem M, Aldahmash A, Alajez NM (2014) MicroRNAs as Regulators of Adipogenic Differentiation of Mesenchymal Stem Cells Stem Cells and Development 24:417-425

He $\mathrm{H}$ et al. (2018) miR-148a-3p promotes rabbit preadipocyte differentiation by targeting PTEN In Vitro Cellular \& Developmental Biology - Animal 54:241-249

Hunt CR, Ro JH, Dobson DE, Min HY, Spiegelman BM (1986) Adipocyte P2 gene: developmental expression and homology of $5 \backslash$ "-flanking sequences among fat cell-specific genes Proceedings of the National Academy of Sciences 83:3786-3790

Kapsimali M, Kloosterman WP, Bruijn ED, Rosa F, Wilson SW (2007) MicroRNAs show a wide diversity of expression profiles in the developing and mature CNS Genome Biology 8:R173

Lagos-Quintana, M. (2001) Identification of Novel Genes Coding for Small Expressed RNAs Science 294:853-858

Lefterova MI, Lazar MA (2009) New developments in adipogenesis Trends in Endocrinology \& Metabolism 20:107-114

Li G, Ning C, Ma Y, Jin L, Liu H (2017) miR-26b Promotes 3T3-L1 Adipocyte Differentiation Through Targeting PTEN Dna \& Cell Biology 36:672

Li X, L H, Q Y (2017) MiR-9-5p promotes MSC migration by activating $\beta$-catenin signaling pathway American Journal of Physiology-Cell Physiology 313:C80-C93

Matteri R (1998) THE BIOLOGY OF LEPTIN: A REVIEW 76:1405-1420

McClelland GB, Kraft CS, Michaud D, Russell JC, Mueller CR, Moyes CD (2004) Leptin and the control of respiratory gene expression in muscle Biochim Biophys Acta 1688:86-93 
Moitra J, Mason MM, Olive M, Krylov D, Vinson C (1998) Life without white fat: a transgenic mouse Genes \& Development 12:3168-3181

MOLDES M, ZUO Y, MORRISON RF, SILVA D, PARK B-H, LIU J, FARMER SR (2003) Peroxisome-proliferatoractivated receptor $\gamma$ suppresses Wnt/ $\beta$-catenin signalling during adipogenesis Biochemical Journal 376:607-613

Papaspyrou-Rao S, Schneider SH, Petersen RN, Fried SK (1997) Dexamethasone Increases Leptin Expression in Humans in Vivo J Clin Endocrinol Metab 82:1635-1637

Picard F et al. (2004) Sirt1 promotes fat mobilization in white adipocytes by repressing PPARy Nature 429:771-776

Plaisance V, Abderrahmani A, Perret-Menoud V (2006a) MicroRNA-9 controls the expression of Granuphilin/SIp4 and the secretory response of insulin-producing cells Journal of Biological Chemistry 281:26932-26942

Plaisance V, Abderrahmani A, Perret-Menoud V, Jacquemin P, Lemaigre F, Regazzi R (2006b) MicroRNA-9 Controls the Expression of Granuphilin/SIp4 and the Secretory Response of Insulin-producing Cells Journal of Biological Chemistry 281:26932-26942

Prayitno DS, Phillips CJC, Omed H (1997) The Effects of Color of Lighting on the Behavior and Production of Meat Chickens Poultry Science 76:452-457

Qi F, Hu JF, Liu BH, Wu CQ, Yu HY, Yao DK, Zhu L (2015) Mi R-9a-5p regulates proliferation and migration of hepatic stellate cells under pressure through inhibition of Sirt1 World Journal of Gastroenterology 21:82-97

Ramachandran D, Roy U, Garg S, Ghosh S, Pathak S, Kolthur-Seetharam U (2011) Sirt1 and mir-9 expression is regulated during glucose-stimulated insulin secretion in pancreatic $\beta$-islets Febs Journal 278:1167-1174

Ross SE, Hemati N, Longo Ka, Bennett CN, Lucas PC, Erickson RL, MacDougald Oa (2000) Inhibition of adipogenesis by Wnt signaling Science 289:950-953

Sanigorski A, Cameron-Smith D, Lewandowski P, Walder K, Silva Ad, Morton G, Collier GR (2013) Impact of obesity and leptin treatment on adipocyte gene expression in Psammomys obesus Journal of Endocrinology 164:45-50

Sato K, NAKANISHI N, MITSUMOTO M (1996) Culture Conditions Supporting Adipose Conversion of Stromal-Vascular Cells from Bovine Intramuscular Adipose Tissues Journal of Veterinary Medical Science 58:1073-1078 
Shimabukuro M et al. (1997) Direct antidiabetic effect of leptin through triglyceride depletion of tissues Proceedings of the National Academy of Sciences of the United States of America 94:4637-4641

Taniguchi M, Nakajima I, Chikuni K, Kojima M, Mikawa S (2014) MicroRNA-33b downregulates the differentiation and development of porcine preadipocytes Molecular Biology Reports 41:1081-1090

Thierry T, Francesca G, Sundeep K, D. JM, Bartolome B, Lawrence RB (1999) Leptin Acts on Human Marrow Stromal Cells to Enhance Differentiation to Osteoblasts and to Inhibit Differentiation to Adipocytes* Endocrinology 140 1630-1638

Wang J, Obici S, Morgan K (2001) Overfeeding Rapidly Induces Leptin and Insulin Resistance Diabetes 50:2786-2791

Warner RD, Greenwood PL, Pethick DW, Ferguson DM (2010) Genetic and environmental effects on meat quality Meat Science 86:171-183

Warriss PD (1999) Meat science: an introductory text International Journal of Food Science \& Technology $36: 449-449$

Wienholds, E. MicroRNA Expression in Zebrafish Embryonic Development Science 309:310-311

Wood JD et al. (2008) Fat deposition, fatty acid composition and meat quality: A review Meat Science 78:343-358

Xie X, Song J, Li G (2016) MiR-21a-5p suppresses bisphenol A-induced pre-adipocyte differentiation by targeting map2k3 through MKK3/p38/MAPK Biochemical and Biophysical Research Communications 473:140-146

Xu P, Y VS, M G (2003) The Drosophila microRNA Mir-14 suppresses cell death and is required for normal fat metabolism Current Biology 13:790-795

Zhang, S. (1996) Obese Gene Expression Alters the Ability of 30A5 Preadipocytes to Respond to Lipogenic Hormones Journal of Biological Chemistry 271:13939-13942

Zhang WR et al. (2017) miR-143 regulates proliferation and differentiation of bovine skeletal muscle satellite cells by targeting IGFBP5 In Vitro Cell Dev Biol Anim 53 265-271

Zhao W, Yang H, Li J, Chen Y, Zhang H (2018) MiR-183 promotes preadipocyte differentiation by suppressing Smad4 in goats Gene 666:158-164

Zhou Y-T, Wang Z-W, Higa M, Newgard CB, Unger RH (1999) Reversing Adipocyte Differentiation: Implications for Treatment of Obesity Proceedings of the National Academy of Sciences of the United States of America 96:2391-2395 


\section{Figures}

\section{Figure 1}

Rabbit preadipocyte differentiation model (A) Oil Red-O staining of lipid droplets ; (B) quantitative detection of oil red $\mathrm{O}$ staining; (C) MiR-9-5p expression levels during preadipocyte differentiation under normal culture conditions; (D) FABP4 expression levels during preadipocyte differentiation under normal culture conditions; (E) CEBPa mRNA expression levels during preadipocyte differentiation under normal culture conditions; (F) PPARy expression levels during preadipocyte differentiation under normal culture

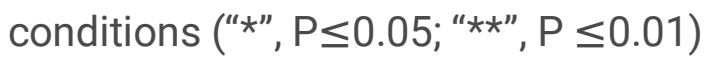
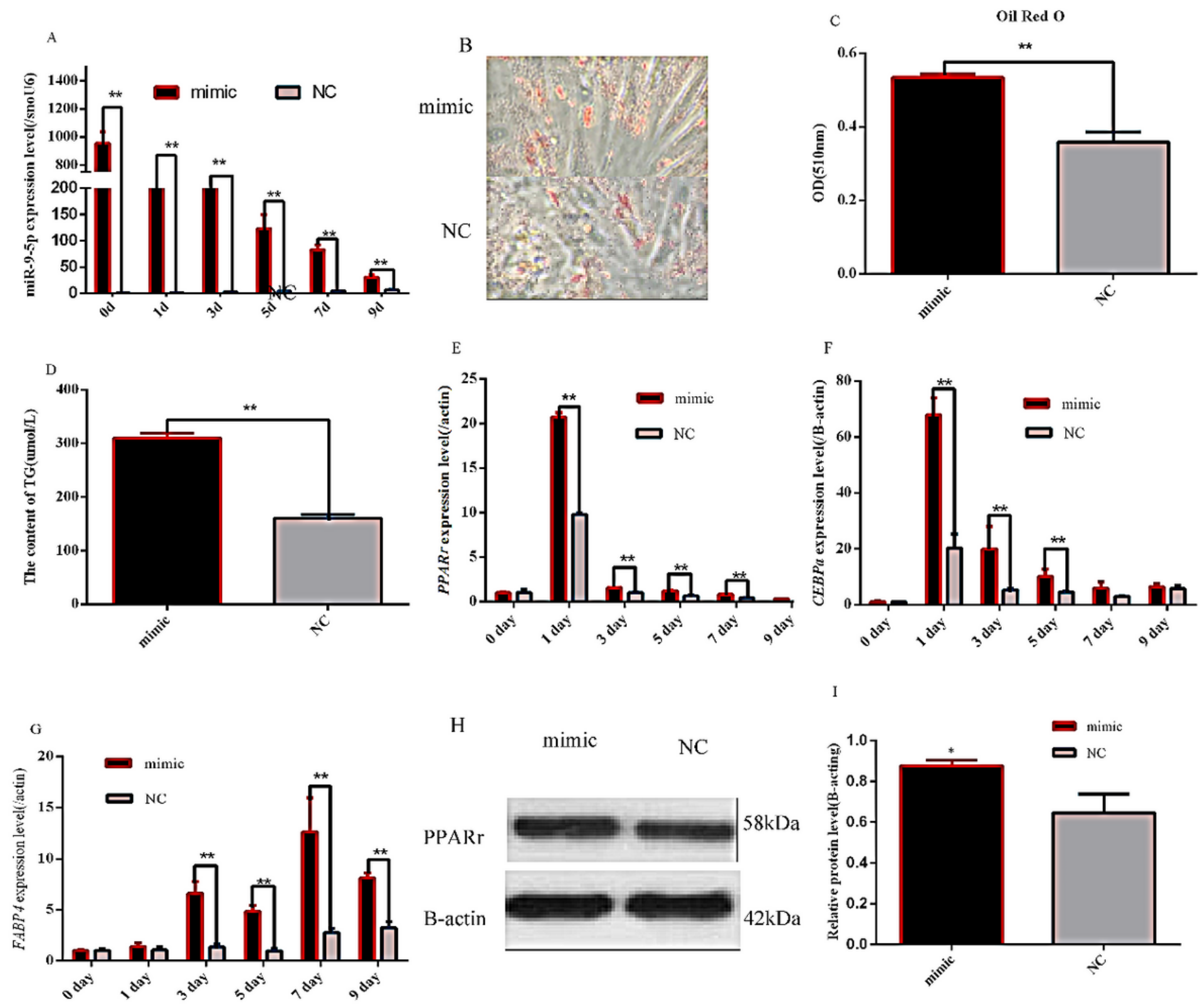

Figure 2 
Overexpression of miR-9-5p promoted rabbit preadipocyte differentiation (A) MiR-9-5p expression levels during preadipocyte differentiation after transfecting with miR-9-5p mimics and NC, (B) Oil Red-O staining of lipid droplets on day 9; (C) Quantitative detection of oil red O staining on day 9; (D) Accumulation of triacylglycerol on day 9; (E) PPARy expression levels during preadipocyte differentiation after transfecting with miR-9-5p mimics and NC; (F) CEBPa expression levels during preadipocyte differentiation after transfecting with miR-9-5p mimics and NC; (G) FABP4 expression levels during preadipocyte differentiation after transfecting with miR-9-5p mimics and NC $(H, I)$ PPARy protein levels during

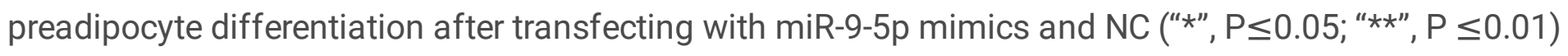

A
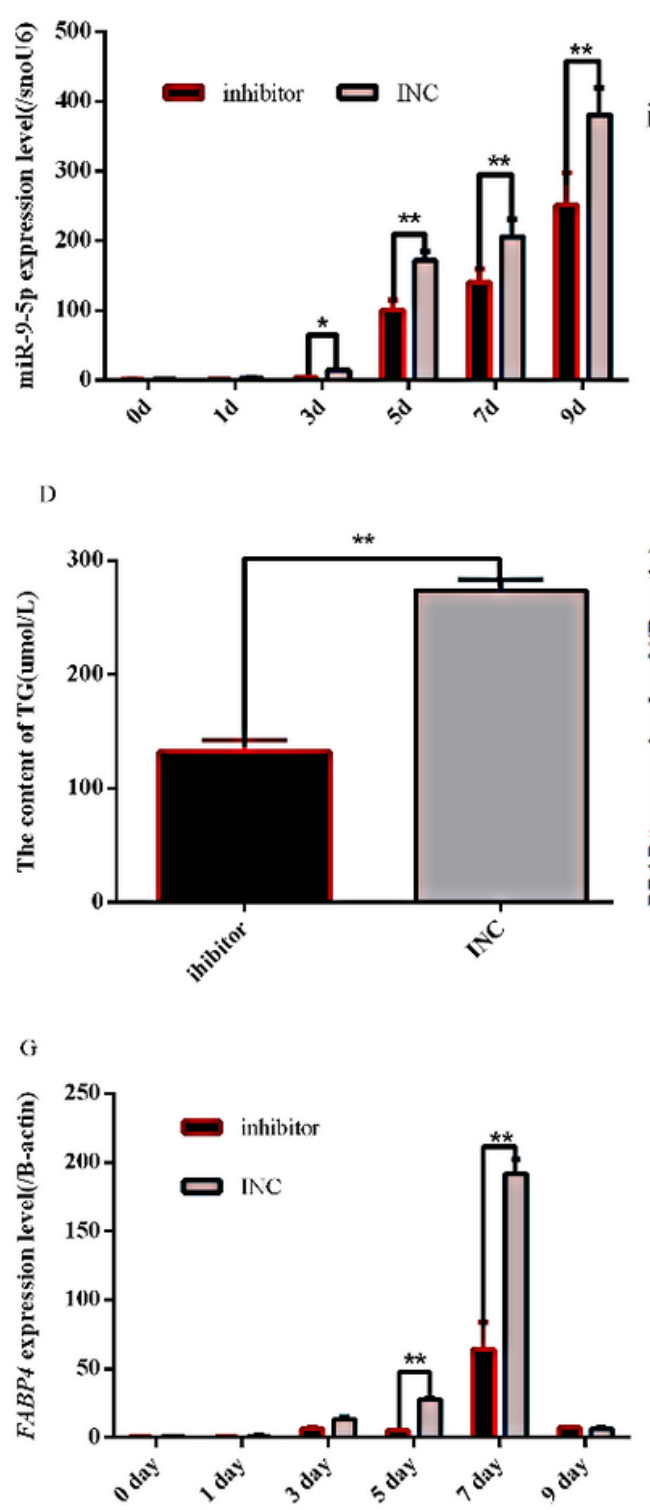

B
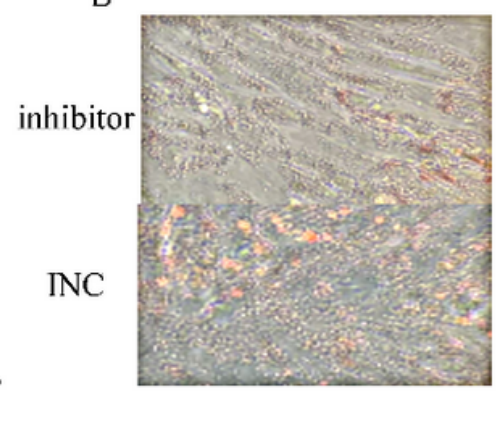

E

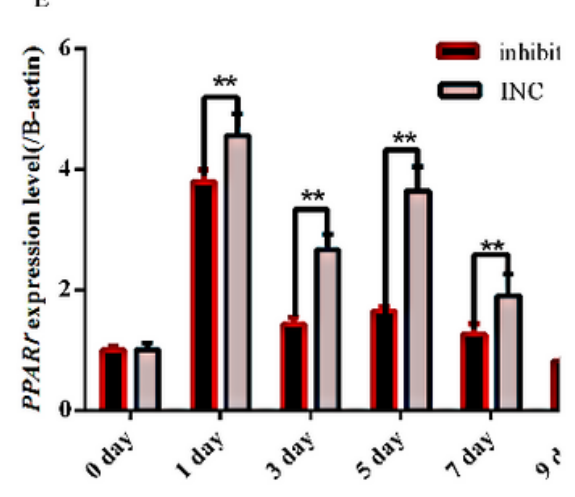

$\mathrm{H}$

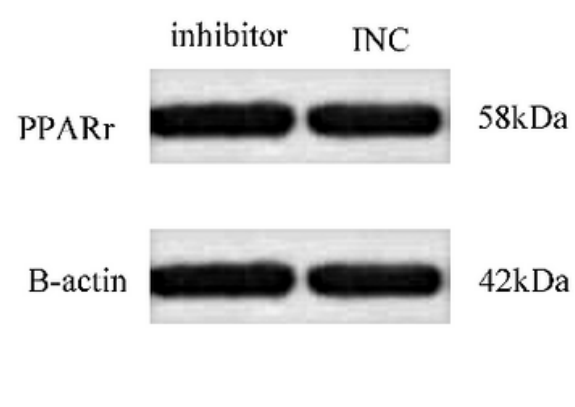

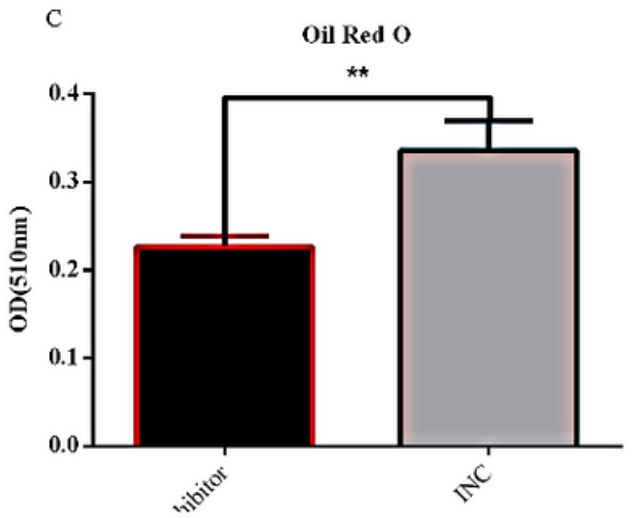

F
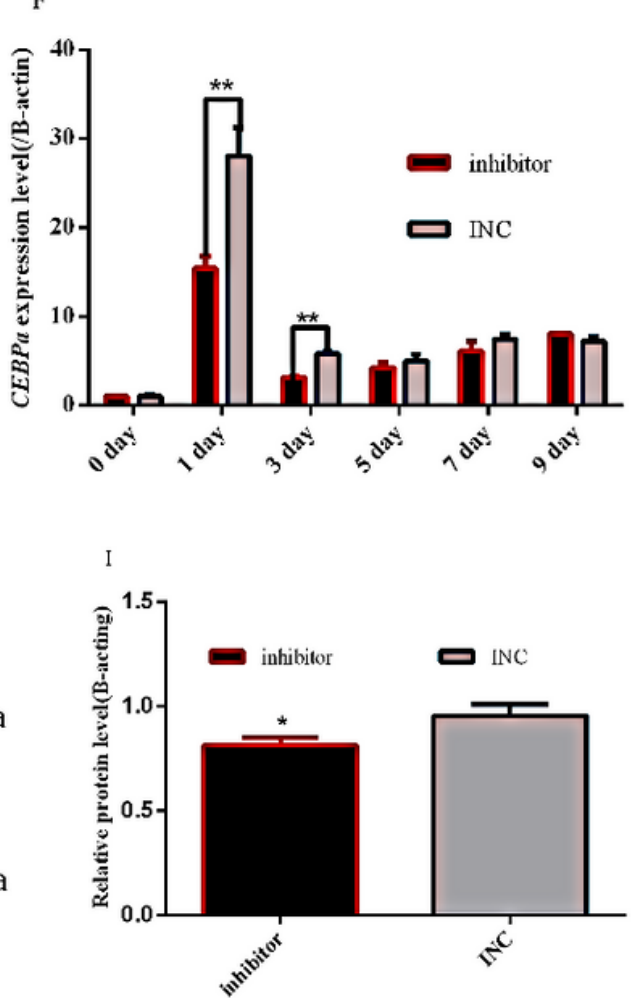

Figure 3

Inhibition of miR-9-5p inhibited rabbit preadipocyte differentiation (A) MiR-9-5p expression levels during preadipocyte differentiation after transfecting with the miR-9-5p inhibitor and INC, (B) Oil Red-O staining of lipid droplets on day 9; (C) Quantitative detection of oil red O staining on day 9; (D) Accumulation of 
triacylglycerol on day 9; (E) PPARy expression levels during preadipocyte differentiation after transfecting with the miR-9-5p inhibitor and INC; (F) CEBPa expression levels during preadipocyte differentiation after transfecting with the miR-9-5p inhibitor and INC; (G) FABP4 expression levels during preadipocyte differentiation after transfecting with the miR-9-5p inhibitor and INC $(H, I)$ PPARY protein levels during

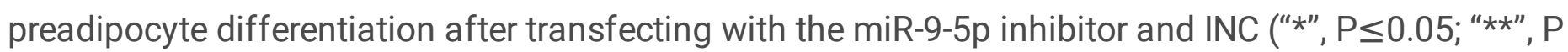
$\leq 0.01)$

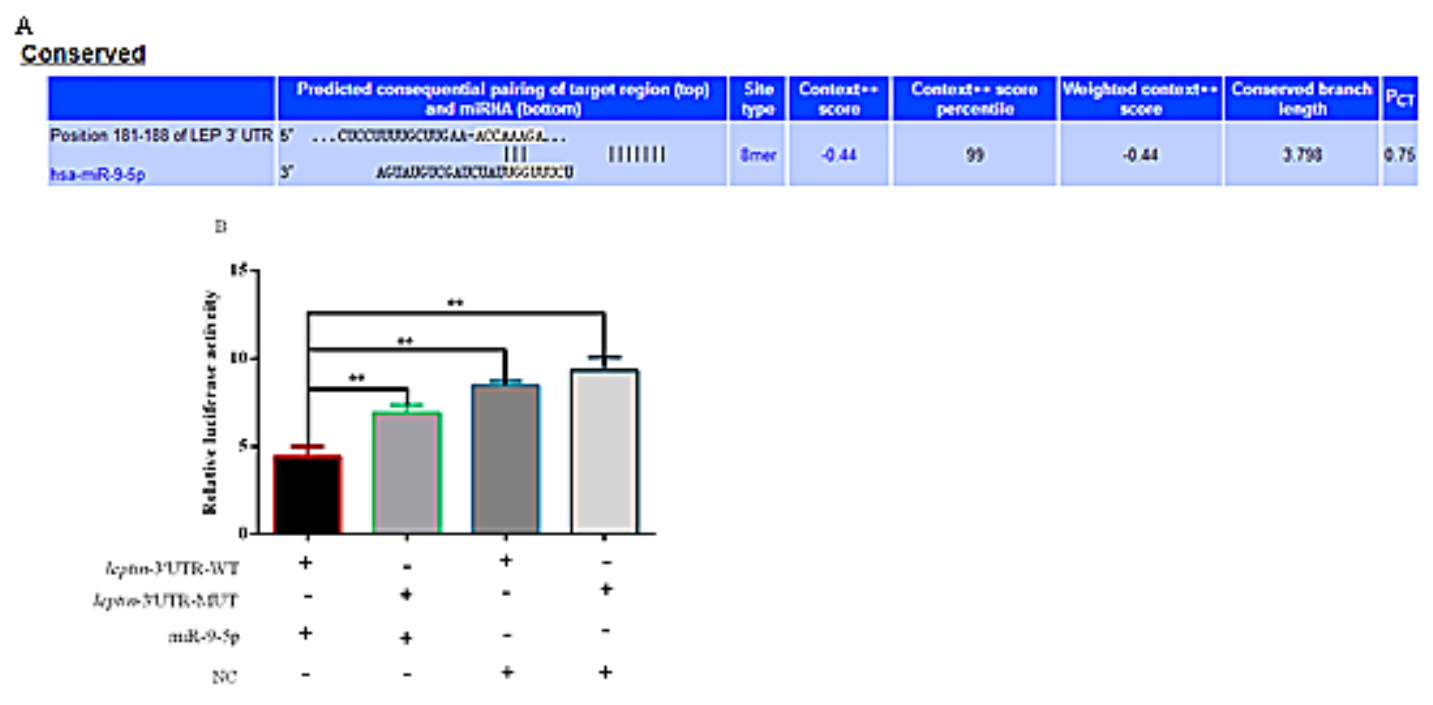

\section{Figure 4}

Leptin is a target miR-9-5p gene. (A) prediction of target gene (B) PmirGLO and pmirGLO-leptin 3' UTR were cotransfected with the mimic into Hela cells, and normalized firefly luciferase activity was determined $(n=3)$. (** $P<0.01)$.
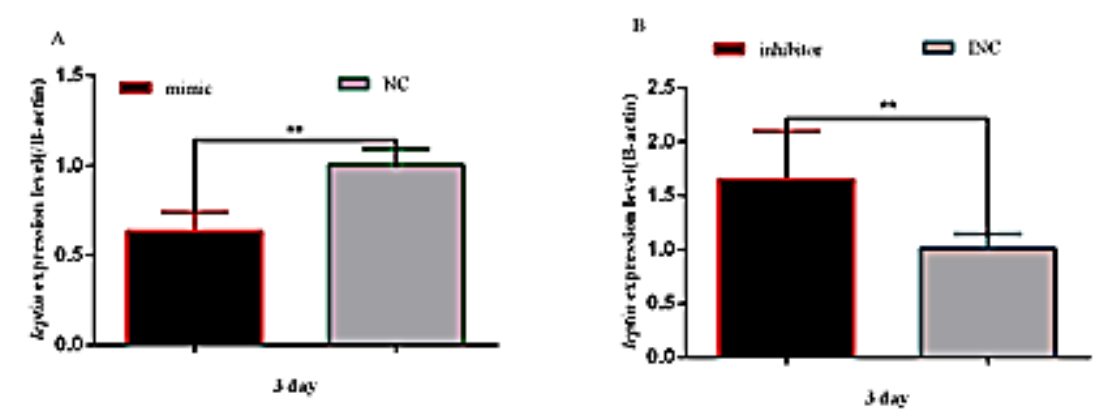

Figure 5

MiR-9-5p negatively regulated leptin expression: (A) Leptin expression based on quantitative real-time polymerase chain reaction (QRT-PCR) during adipogenesis with miR-9-5p overexpression. (B)Leptin

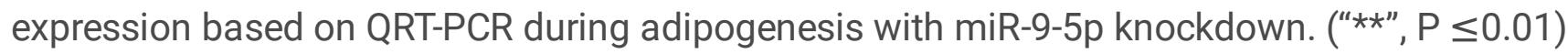



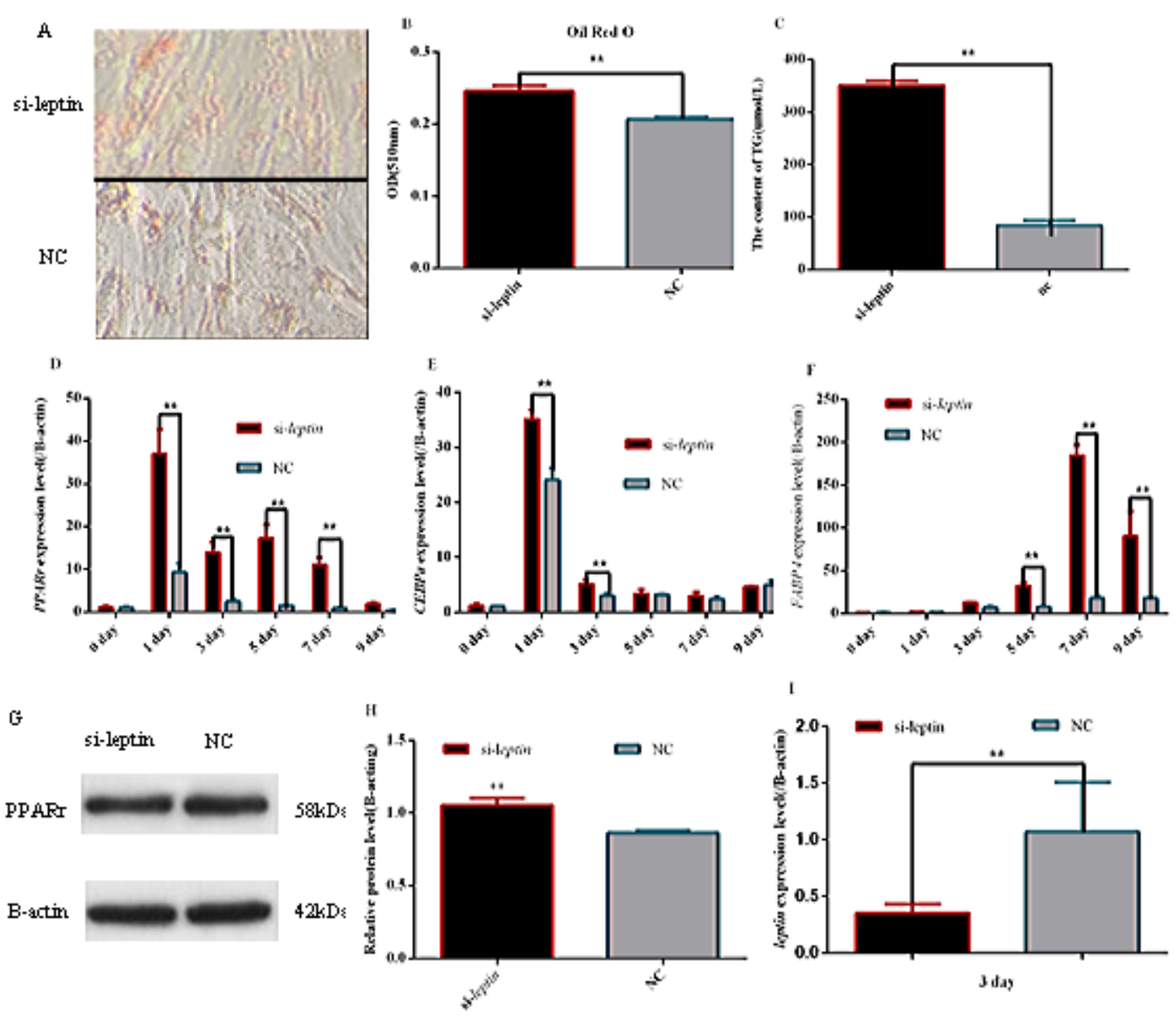

Figure 6

Inhibition of leptin gene promoted rabbit preadipocyte differentiation (A) Oil Red-O staining of lipid droplets on day 9; (B) Quantitative detection of oil red O staining on day 9; (C) Accumulation of triacylglycerol on day 9; (D) PPARy expression levels during preadipocyte differentiation after transfecting with si-leptin and NC; (E) CEBPa expression levels during preadipocyte differentiation after transfecting with si-leptin and NC; (F) FABP4 expression levels during preadipocyte differentiation after transfecting with si-leptin and NC; $(\mathrm{G}, \mathrm{H})$ PPARy protein levels during preadipocyte differentiation after transfecting with si-leptin and NC. (I) Leptin. expression levels during preadipocyte differentiation after transfecting

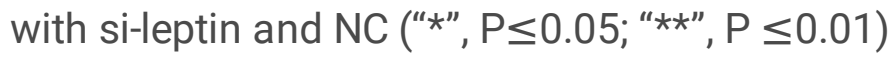

